Global Journal of Pure and Applied Mathematics.

ISSN 0973-1768 Volume 16, Number 6 (2020), pp. 915-937

(C) Research India Publications

https://dx.doi.org/10.37622/GJPAM/16.6.2020.915-937

\title{
Models of Prey-Predator Systems with Two Mutualistic Predators
}

\author{
Yogendra Singh and Ravinder Kumar \\ Department of Mathematics, Dayalbagh Educational Institute (Deemed University), \\ Dayalbagh, Agra - 282005, Uttar Pradesh, India
}

\begin{abstract}
Models of population's interaction among one or two preys and two predator mutualists are studied. Sufficient conditions for local and global stability of the equilibria and uniform persistence are presented.
\end{abstract}

\section{Introduction}

In this paper we study models involving one or two preys and two mutualistic predators. We study both facultative and obligate mutualism. An example of this type of mutualism occurs in Red Sea [3,8], where the effect of mutualism is to increase the predator functional response. There, Yellow saddle goatfish, P. cyclostouces, and bird wrasse, Gomphosus caeruleus, tackle coral reefs from both sides so that the prey may be driven toward each other and thereby caught and eaten. Without the cooperation between these two fish species, such prey would be available only minimally and with great difficulty. Hence, the effect of each predator population on the other is to increase hunting efficiency. In earlier studies such three species models are analyzed in $[3,6]$. Specifically sufficient conditions for uniform persistence are obtained. Other models involving mutualism are studied in $([7,9,10]$ and references theirin).

In the next section we describe our model. We show that the model is meaningful, i.e. solutions with positive initial conditions stay positive and are bounded in forward time. In section 3 we consider existence of equilibria in case of facultative and 
obligate mutualism and study their local stability. In section 4 we derive conditions for global asymptotic stability of interior equilibria of three/four dimensional subsystems/system. In section 5 we obtain sufficient conditions for uniform persistence. In the section 6 we study a special case of our model and present numerical examples to illustrate our results.

\section{The Model}

The model is:

$\frac{d x_{1}}{d t}=x_{1} g_{1}\left(x_{1}\right)-y_{1} p_{1}\left(x_{1}, y_{2}\right)-y_{2} q_{1}\left(x_{1}, y_{1}\right)$

$\frac{d x_{2}}{d t}=x_{2} g_{2}\left(x_{2}\right)-y_{1} p_{2}\left(x_{2}, y_{2}\right)-y_{2} q_{2}\left(x_{2}, y_{1}\right)$,

$\frac{d y_{1}}{d t}=y_{1}\left[-s_{1}\left(y_{1}\right)+c_{1} p_{1}\left(x_{1}, y_{2}\right)+\widetilde{c_{1}} p_{2}\left(x_{2}, y_{2}\right)\right]$

$\frac{d y_{2}}{d t}=y_{2}\left[-s_{2}\left(y_{2}\right)+c_{2} q_{1}\left(x_{1}, y_{1}\right)+\widetilde{c_{2}} q_{2}\left(x_{2}, y_{1}\right)\right]$,

$x_{i}(0)=x_{i 0} \geq 0, y_{i}(0)=y_{i 0} \geq 0, i=1,2$,

where the variables $x_{1}, x_{2}$ denote prey densities and $y_{1}, y_{2}$ that of predators.

We assume the following hypotheses on the given functions,

$\left(H_{1}\right)$ all functions are continuously differentiable so that solution to I.V.P. (1) exist, are unique and can be continued for all positive time.

$\left(H_{2}\right) g_{i}\left(x_{i}\right):[0, \infty) \rightarrow R, g_{i}(0)>0, g_{i}^{\prime}<0$; There exist $K_{i}^{\prime}$ 's such that $g_{i}\left(K_{i}\right)=$ $0, i=1,2$. The constants $K_{1}$ and $K_{2}$ are the carrying capacities of $x_{1}$ and $x_{2}$ respectively.

The functions $p_{i}\left(x_{i}, y_{2}\right), i=1,2$ are predator response functions of the predator $y_{1}$. We assume

$\left(H_{3}\right) p_{i}\left(0, y_{2}\right)=0, \frac{\partial p_{i}}{\partial x_{i}} \geq 0, \frac{\partial p_{i}}{\partial y_{2}} \geq 0, i=1,2$.

These conditions imply that there is no predation in absence of prey and that the predator response function $p_{i}\left(x_{i}, y_{2}\right)$ is an increasing function of density $x_{i}$. This hypothesis implies that $y_{2}$ increases the predation by $y_{1}$.

We also assume

$\left(H_{4}\right) q_{i}\left(0, y_{1}\right)=0, \quad \frac{\partial q_{i}}{\partial x_{i}} \geq 0, \quad$ and $\frac{\partial q_{i}}{\partial y_{1}} \geq 0, \quad i=1,2$.

The positive constants rate of conversion $c_{i}$ and $\widetilde{c_{l}}$ denote the rate of conversion of 
prey biomass to predator biomass.

$\left(H_{5}\right)$ Finally $s_{i}\left(y_{i}\right)$ denotes the death rate of the predator $y_{i}$ and $\frac{\partial s_{i}}{\partial y_{i}}>0, i=1,2$.

The first result is:

Theorem 1: The solutions to IVP (1) with positive initial conditions stay positive for $t>0$.

Proof - We rewrite first equation in (1) as,

$\int_{0}^{t} \frac{d x_{1}}{x_{1}}=\int_{0}^{t}\left\{g_{1}\left(x_{1}(s)\right)-y_{1}(s) \frac{p_{1}\left(x_{1}(s), y_{2}(s)\right)}{x_{1}(s)}-y_{2}(s) \frac{q_{1}\left(x_{1}(s), y_{1}(s)\right)}{x_{1}(s)}\right\} d s$,

where, $\lim _{x_{1} \rightarrow 0} \frac{p_{1}\left(x_{1}, y_{2}\right)}{x_{1}}=\frac{\partial p_{1}\left(0, y_{2}\right)}{\partial x_{1}}>0$, and $\lim _{x_{1} \rightarrow 0} \frac{q_{1}\left(x_{1}, y_{1}\right)}{x_{1}}=\frac{\partial q_{1}\left(0, y_{1}\right)}{\partial x_{1}}>0$.

Thus, $x_{1}(t)=x_{10} e^{\int_{0}^{t}\left(g_{1}-\frac{y_{1} p_{1}}{x_{1}}-\frac{y_{2} q_{1}}{x_{1}}\right) d s}>0$

Proceeding similarly we can show $x_{2}(t)>0, y_{1}(t)>0$ and $y_{2}(t)>0$ for all $t>0$.

Theorem 2: Let $G_{i}=\max _{\left[0, K_{i}\right]}\left(s_{1}(0)+g_{i}(x)\right) x, i=1,2, L_{i}=\max _{\left[0, K_{i}\right]}\left(s_{2}(0)+g_{i}(x)\right) x$, $i=1,2$.

Further let,

$\mathcal{A}=\left\{\left(x_{1}, x_{2}, y_{1}, y_{2}\right): 0 \leq x_{1} \leq K_{1}, 0 \leq x_{1} \leq K_{2}, 0 \leq c_{1} x_{1}+\widetilde{c_{1}} x_{2}+y_{1} \leq\right.$

$\left.\frac{c_{1} G_{1}+\widetilde{c_{1}} G_{2}}{s_{1}(0)}, 0 \leq c_{2} x_{1}+\widetilde{c_{2}} x_{2}+y_{2} \leq \frac{c_{2} L_{1}+\widetilde{c_{2}} L_{2}}{s_{2}(0)}\right\}$.

Then

(i) $\mathcal{A}$ is positively invariant.

(ii) $\quad\left(x_{1}, x_{2}, y_{1}, y_{2}\right) \rightarrow \mathcal{A}$ as $t \rightarrow \infty$

Proof - Let $0 \leq x_{1}(0) \leq K_{1}$. Then $u^{\prime}=u g_{1}(u), u(0)=x_{1}(0)$, has solution $u(t) \leq$ $K_{1}$. Thus by comparison Theorem [ 5 ] $x_{1}(t) \leq K_{1}$. In general, $\lim \sup _{t \rightarrow \infty} x_{1}(t) \leq$ $K_{1}$

\section{Similarly}

$0 \leq x_{2}(0) \leq K_{2}$ implies, $x_{2}(t) \leq K_{2}$. In general, lim sup $\operatorname{su\infty }_{t \rightarrow \infty} x_{2}(t) \leq K_{2}$.

Next ,

$$
\begin{aligned}
\left(c_{1} x_{1}+\widetilde{c_{1}} x_{2}\right. & \left.+y_{1}\right)^{\prime} \\
& \leq c_{1} x_{1} g_{1}\left(x_{1}\right)+x_{2} \widetilde{c_{1}} g_{2}\left(x_{2}\right)-s_{1}(0) y_{1}-c_{1} x_{1} s_{1}(0)-x_{2} \widetilde{c_{1}} s_{1}(0) \\
& +c_{1} x_{1} s_{1}(0)+\widetilde{c_{1}} x_{2} s_{1}(0) .
\end{aligned}
$$


Set $u=c_{1} x_{1}+\widetilde{c_{1}} x_{2}+y_{1}$ to get,

$$
\begin{gathered}
u^{\prime}+s_{1}(0) u \leq c_{1} x_{1}\left(s_{1}(0)+g_{1}\left(x_{1}\right)\right)+\widetilde{c_{1}} x_{2}\left(s_{1}(0)+g_{2}\left(x_{2}\right)\right) \\
\leq c_{1} G_{1}+\widetilde{c_{1}} G_{2}
\end{gathered}
$$

Solving for $u(t)$ and using comparison theorem,

$\left(u e^{s_{1}(0) t}-u(0)\right) \leq \frac{\left(c_{1} G_{1}+\widetilde{c_{1}} G_{2}\right)\left(e^{s_{1}(0) t}-1\right)}{s_{1}(0)}$,

Or $u(t) \leq u(0) e^{-s_{1}(0) t}+\frac{\left(C_{1} G_{1}+\widetilde{c_{1}} G_{2}\right)\left(1-e^{s_{1}(0) t}\right)}{s_{1}(0)}$,

$u(t) \leq e^{-s_{1}}(0) t\left(u(0)-\frac{c_{1} G_{1}+\widetilde{c_{1}} G_{2}}{s_{1}(0)}\right)+\frac{c_{1} G_{1}+\widetilde{c_{1}} G_{2}}{s_{1}(0)}$.

So, $c_{1} x_{1}(0)+\widetilde{c_{1}} x_{2}(0)+y_{1}(0) \leq \frac{c_{1} G_{1}+\widetilde{c_{1}} G_{2}}{s_{1}(0)}$

Implies, $c_{1} x_{1}+\widetilde{c_{1}} x_{2}+y_{1} \leq \frac{c_{1} G_{1}+\widetilde{c_{1}} G_{2}}{s_{1}(0)}$.

In general,

$c_{1} x_{1}(t)+\widetilde{c_{1}} x_{2}(t)+y_{1}(t) \leq \frac{c_{1} G_{1}+\widetilde{c_{1}} G_{2}}{s_{1}(0)}+\epsilon$, as $t \rightarrow \infty$.

Similar argument holds for $c_{2} x_{1}+\widetilde{c_{2}} x_{2}+y_{2}$.

\section{Equilibria and their local stability}

In this section we study existence of equilibria depending on the form of mutualism.

\subsubsection{The Facultative Mutualism}

In this section we consider the case when predators exhibit facultative mutualism, i.e. when both predator populations are capable of surviving on their own, but are able to sustain higher population numbers due to mutualism. Thus we require, $\exists \bar{x}_{l}^{*}$ such that,

$$
\begin{gathered}
-s_{1}(0)+c_{1} p_{1}\left({\overline{x_{1}}}^{*}, 0\right)=0, \\
-s_{1}(0)+\widetilde{c_{1}} p_{2}\left({\overline{x_{2}}}^{*}, 0\right)=0, \\
{\overline{x_{l}}}^{*}<K_{i}, \quad i=1,2 .
\end{gathered}
$$

If (3.1) does not hold, there exists no equilibrium in positive $x_{1}-y_{1}$ quadrant and hence has no periodic solution in it. Hence by Poincare Bendixson Theorem, equilibrium $\left(K_{1}, 0,0,0\right)$ is globally asymptotically stable in the positive $x_{1}-y_{1}$ quadrant. Similar conclusion follows when (3.2) does not hold.

Similarly, we require 


$$
\begin{aligned}
& -s_{2}(0)+c_{2} q_{1}\left(\widetilde{x_{1}}{ }^{*}, 0\right)=0, \\
& -s_{2}(0)+\widetilde{c_{2}} q_{2}\left(\widetilde{x_{2}}, 0\right)=0, \\
& \text { for some }{\widetilde{x_{l}}}^{*}<K_{i}, i=1,2 .
\end{aligned}
$$

We conclude, that there exist equilibria

$E_{0}(0,0,0,0), E_{1}\left(K_{1}, 0,0,0\right), E_{2}\left(0, K_{2}, 0,0\right), E_{3}\left(\overline{x_{1}}, 0, \overline{y_{1}}, 0\right), E_{4}\left(0, \overline{x_{2}}, \overline{y_{11}}, 0\right)$, $E_{5}\left(\widetilde{x_{1}}, 0,0, \widetilde{y_{2}}\right), E_{6}\left(0, \widetilde{x_{2}}, 0, \widetilde{y_{22}}\right), E_{7}\left(x_{31}, x_{32}, y_{31}, 0\right), E_{8}\left(x_{41}, x_{42}, 0, y_{42}\right), E_{9}\left(x_{51}, 0, y_{51}, y_{52}\right)$ and $E_{10}\left(0, x_{62}, y_{61}, y_{62}\right)$. Also interior equilibrium $E^{*}\left(x_{1}^{*}, x_{2}^{*}, y_{1}^{*}, y_{2}^{*}\right)$ may exist. One sufficient condition for existence of $E^{*}$ is that system (1) be uniformly persistent (see[ $2])$.

\subsubsection{Facultative - Obligate mutualism}

Next we consider the case when mutualism is obligate for one predator and facultative for the other. The mutualism will be obligate for $y_{1}$, when

$$
-s_{1}(0)+c_{1} p_{1}\left(K_{1}, 0\right)+\widetilde{c_{1}} p_{2}\left(K_{2}, 0\right)<0
$$

It will be obligate for $y_{2}$, when

$-s_{2}(0)+c_{2} q_{1}\left(K_{1}, 0\right)+\widetilde{c_{2}} q_{2}\left(K_{2}, 0\right)<0$

When (5.1) holds then boundary equilibria $E_{3}, E_{4}$ and $E_{7}$ do not exist.

When (5.2) holds then equilibria $E_{5}, E_{6}$ and $E_{8}$ do not exist.

\subsubsection{Obligate Mutualism}

When (5.1) and (5.2) both hold then mutualism is obligate for both $y_{1}$ and $y_{2}$. In this case equilibria $E_{3}, E_{4}, E_{5}, E_{6}, E_{7}$ and $E_{8}$ do not exist.

\subsection{Stability of Equilibria}

Jacobian matrix $V$ of system (1) is,

$V=\left[\begin{array}{cccc}g_{1}+x_{1} g_{1}^{\prime}-y_{1} p_{1 x_{1}}-y_{2} q_{1 x_{1}} & 0 & -p_{1}-y_{2} q_{1 y_{1}} & -y_{1} p_{1 y_{2}}-q_{1} \\ 0 & g_{2}+x_{2} g_{2}^{\prime}-y_{1} p_{2 x_{2}}-y_{2} q_{2 x_{2}} & -p_{2}-y_{2} q_{2 y_{1}} & -y_{1} p_{2 y_{2}}-q_{2} \\ y_{1} c_{1} p_{1 x_{1}} & y_{1} \widetilde{1_{1}} p_{2 x_{2}} & -s_{1}+c_{1} p_{1}+\widetilde{c_{1}} p_{2}-y_{1} s_{1}^{\prime} & c_{1} y_{1} p_{1 y_{2}}+\widetilde{c_{1}} p_{2 y_{2}} \\ c_{2} y_{2} q_{1 x_{1}} & \widetilde{c_{2}} y_{2} q_{2 x_{2}} & c_{2} y_{2} q_{1 y_{1}}+\widetilde{c_{2}} q_{2 y_{1}} y_{2} & -s_{2}+c_{2} q_{1}+\widetilde{c_{2}} q_{2}-y_{2} s_{2}^{\prime}\end{array}\right]$

$V\left(E_{0}\right)=\operatorname{diag}\left(g_{1}(0), g_{2}(0),-s_{1}(0),-s_{2}(0)\right)$

So $E_{0}$ is unstable in $x_{1}$ and $x_{2}$ directions and stable in $y_{1}$ and $y_{2}$ directions. Next,

$V\left(E_{1}\right)=\left[\begin{array}{cccc}K_{1} g_{1}^{\prime}\left(K_{1}\right) & 0 & -p_{1}\left(K_{1}, 0\right) & -q_{1}\left(K_{1}, 0\right) \\ 0 & g_{2}(0) & 0 & 0 \\ 0 & 0 & -s_{1}(0)+c_{1} p_{1}\left(K_{1}, 0\right) & 0 \\ 0 & 0 & 0 & -s_{2}(0)+c_{2} q_{1}\left(K_{1}, 0\right)\end{array}\right]$

The equilibrium $E_{1}\left(K_{1}, 0,0,0\right)$ is unstable in $x_{2}$ direction. In $y_{1}$ and $y_{2}$ directions the eigenvalues are 
$-s_{1}(0)+c_{1} p_{1}\left(K_{1}, 0\right)$ and $-s_{2}(0)+c_{2} q_{1}\left(K_{1}, 0\right)>0$, respectively.

Same way $E_{2}\left(0, K_{2}, 0,0\right)$ is unstable in $x_{1}$ direction. The eigenvalues of $V\left(E_{2}\right)$ in $y_{1}$ and $y_{2}$ direction are $-s_{1}(0)+\widetilde{c_{1}} p_{2}\left(K_{2}, 0\right)$ and $-s_{2}(0)+\widetilde{c_{2}} q_{2}\left(K_{2}, 0\right)$. respectively. Also,

$V\left[E_{3}\left(\overline{x_{1}}, 0, \overline{y_{1}}, 0\right)\right]=\left[\begin{array}{cccc}g_{1}\left(\overline{x_{1}}\right)+\overline{x_{1}} g_{1}^{\prime}\left(\overline{x_{1}}\right)-\overline{y_{1}} p_{1 x_{1}}\left(\overline{x_{1}}, 0\right) & 0 & -p_{1}\left(\overline{x_{1}}, 0\right) & -\overline{y_{1}} p_{1 y_{2}}\left(\overline{x_{1}}, 0\right)-q_{1}\left(\overline{x_{1}}, \overline{y_{1}}\right) \\ 0 & g_{2}(0)-\overline{y_{1}} p_{2 x_{2}}(0,0) & 0 & 0 \\ c_{1} \overline{y_{1}} p_{1 x_{1}}\left(\overline{x_{1}}, 0\right) & 0 & -s_{1}^{\prime}\left(\overline{y_{1}}\right) \overline{y_{1}} & c_{1} \overline{y_{1}} p_{1 y_{2}}\left(\overline{x_{1}}, 0\right) \\ 0 & 0 & 0 & -s_{2}(0)+c_{2} q_{1}\left(\overline{x_{1}}, \overline{y_{1}}\right)\end{array}\right]$

So $V\left(E_{3}\right)$ has eigenvalue $g_{2}(0)-\overline{y_{1}} p_{2 x_{2}}(0,0)$ in $x_{2}$ - direction. The eigenvalue in $y_{2}$ - direction is $-s_{2}(0)+c_{2} q_{1}\left(\overline{x_{1}}, \overline{y_{1}}\right)$. The other two eigenvalues are roots of $\lambda^{2}+\left(\alpha_{1}+\overline{y_{1}} s_{1}^{\prime}\left(\overline{y_{1}}\right)\right) \lambda+\alpha_{1} \overline{y_{1}} s_{1}^{\prime}\left(\overline{y_{1}}\right)+c_{1} \overline{y_{1}} p_{1}\left(\overline{x_{1}}, 0\right) p_{1 x_{1}}\left(\overline{x_{1}}, 0\right)=0$,

where, $\alpha_{1}=g_{1}\left(\overline{x_{1}}\right)+\overline{x_{1}} g_{1}^{\prime}\left(\overline{x_{1}}\right)-\overline{y_{1}} p_{1 x_{1}}\left(\overline{x_{1}}, 0\right)$.

Proceeding same way $V\left(E_{4}\left(0, \overline{x_{2}}, \overline{y_{11}}, 0\right)\right)$ has eigenvalues $g_{1}(0)-\overline{y_{11}} p_{1 x_{1}}(0,0)$ and $-s_{2}(0)+\widetilde{c_{2}} q_{2}\left(\overline{x_{2}}, \overline{y_{11}}\right)$ in $x_{1}$ and $y_{2}$ directions, respectively. The other two eigenvalues will be given by,

$\lambda^{2}+\left(\alpha_{2}+\overline{y_{11}} s_{2}^{\prime}\left(\overline{y_{11}}\right)\right) \lambda+\alpha_{2} \overline{y_{11}} s_{1}^{\prime}\left(\overline{y_{11}}\right)+\widetilde{c_{1}} \overline{y_{11}} p_{2}\left(\overline{x_{2}}, 0\right) p_{2 x_{2}}\left(\overline{x_{2}}, 0\right)=0$, where, $\alpha_{2}=g_{2}\left(\overline{x_{2}}\right)+\overline{x_{2}} g_{2}^{\prime}\left(\overline{x_{2}}\right)-\overline{y_{11}} p_{2 x_{2}}\left(\overline{x_{2}}, 0\right)$.

Also, $V\left(E_{5}\left(\widetilde{x_{1}}, 0,0, \widetilde{y_{2}}\right)\right)$ has eigenvalues $g_{2}(0)-\widetilde{y_{2}} q_{2 x_{2}}(0,0)$ and $-s_{1}(0)+$ $c_{2} p_{1}\left(\widetilde{x_{1}}, \widetilde{y_{2}}\right)$ in $x_{2}$ and $y_{1}$ directions. The other two eigenvalues are the roots of $\lambda^{2}+\left(\alpha_{3}+\widetilde{y_{2}} s_{2}^{\prime}\left(\widetilde{y_{2}}\right)\right) \lambda+\alpha_{3} \widetilde{y_{2}} s_{2}^{\prime}\left(\widetilde{y_{2}}\right)+\widetilde{y_{2}} c_{2} q_{1}\left(\widetilde{x_{1}}, 0\right) q_{1 x_{1}}\left(\widetilde{x_{1}}, 0\right)=0$,

where, $\alpha_{3}=g_{1}\left(\widetilde{x_{1}}\right)+\widetilde{x_{1}} g_{1}^{\prime}\left(\widetilde{x_{1}}\right)-\widetilde{y_{2}} q_{1 x_{1}}\left(\widetilde{x_{1}}, 0\right)$.

$V\left(E_{6}\left(0, \widetilde{x_{2}}, 0, \widetilde{y_{22}}\right)\right)$ has eigenvalues $g_{1}(0)-\widetilde{y_{22}} q_{1 x_{1}}(0,0)$ and $-s_{1}(0)+$ $\widetilde{c_{1}} p_{2}\left(\widetilde{x_{2}}, \widetilde{y_{22}}\right)$ in $x_{1}$ and $y_{1}$ directions. The other two eigenvalues are roots of $\lambda^{2}+\left(\alpha_{4}+\widetilde{y_{22}} s_{2}^{\prime}\left(\widetilde{y_{22}}\right)\right) \lambda+\alpha_{4} \widetilde{y_{22}} s_{2}^{\prime}\left(\widetilde{y_{22}}\right)+\widetilde{c_{2}} \widetilde{y_{22}} q_{2}\left(\widetilde{x_{2}}, 0\right) q_{2 x_{2}}\left(\widetilde{x_{2}}, 0\right)=0$, where, $\alpha_{4}=g_{2}\left(\widetilde{x_{2}}\right)+\widetilde{x_{2}} g_{2}^{\prime}\left(\widetilde{x_{2}}\right)-\widetilde{y_{22}} q_{2 x_{2}}\left(\widetilde{x_{2}}, 0\right)$.

Next,

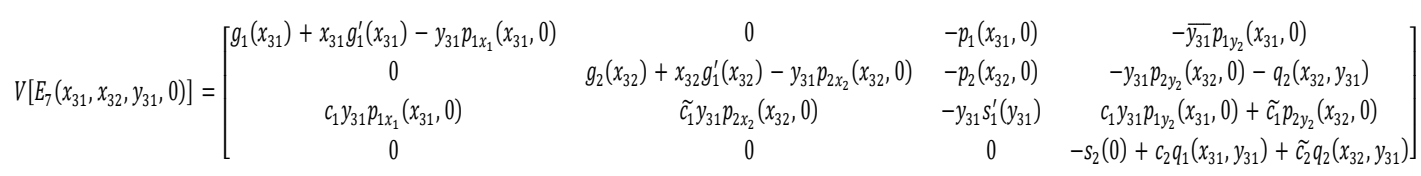

Eigenvalue of $V\left(E_{7}\left(x_{31}, x_{32}, y_{31}, 0\right)\right)$ in $y_{2}$-direction is $\xi_{1}=-s_{2}(0)+c_{2} q_{1}\left(x_{31}, y_{31}\right)+\widetilde{c_{2}} q_{2}\left(x_{32}, y_{31}\right)$. 
Other eigenvalues are the roots of

$\lambda^{3}+\mu_{1} \lambda^{2}+\mu_{2} \lambda+\mu_{3}=0$

where, $\mu_{1}=-\left(y_{31} s_{1}^{\prime}\left(y_{31}\right)+a_{1}+a_{2}\right)$,

$\mu_{2}=a_{3}+a_{1}\left(a_{2}+y_{31} s_{1}^{\prime}\left(y_{31}\right)+c_{1} y_{31} p_{1 x_{1}}\left(x_{31}, 0\right) p_{1}\left(x_{31}, 0\right)\right)$,

$\mu_{3}=-a_{1} a_{3}-a_{2} c_{1} y_{31} p_{1 x_{1}}\left(x_{31}, 0\right) p_{1}\left(x_{31}, 0\right)$,

$a_{i}=g_{i}\left(x_{3 i}\right)+x_{3 i} g_{i}^{\prime}\left(x_{3 i}\right)-y_{31} p_{i x_{i}}\left(x_{3 i}, 0\right), i=1,2$.

and $a_{3}=\widetilde{c_{1}} y_{31} p_{2}\left(x_{32}, 0\right) p_{2 x_{2}}\left(x_{32}, 0\right)-y_{31} s_{1}^{\prime}\left(y_{31}\right) a_{2}$.

Similarly, the eigenvalue of $V\left(E_{8}\left(x_{41}, x_{42}, 0, y_{42}\right)\right)$ in $y_{1}$ - direction is $\xi_{2}=-s_{1}(0)+$ $c_{1} p_{1}\left(x_{41}, y_{42}\right)+\widetilde{c_{1}} p_{2}\left(x_{42}, y_{42}\right)$.

Other eigenvalues are given by the roots of

$\lambda^{3}+\gamma_{1} \lambda^{2}+\gamma_{2} \lambda+\gamma_{3}=0$

$\gamma_{1}=-\left(y_{42} s_{2}^{\prime}\left(y_{42}\right)+a_{4}+a_{5}\right)$

$\gamma_{2}=a_{6}+a_{4}\left(a_{5}+y_{42} s_{2}^{\prime}\left(y_{42}\right)+c_{2} y_{42} q_{1 x_{1}}\left(x_{41} .0\right) q_{1}\left(x_{41}, 0\right)\right)$

$\gamma_{3}=-a_{4} a_{6}-a_{5} c_{2} y_{42} q_{1 x_{1}}\left(x_{41}, 0\right) q_{1}\left(x_{41}, 0\right)$

$a_{i+3}=g_{i}\left(x_{4 i}\right)+x_{4 i} g_{i}^{\prime}\left(x_{4 i}\right)-y_{42} q_{i x_{i}}\left(x_{4 i}, 0\right), i=1,2$,

and $a_{6}=\widetilde{c_{2}} y_{42} q_{2}\left(x_{42}, 0\right) q_{2 x_{2}}\left(x_{42}, 0\right)-y_{42} s_{2}^{\prime}\left(y_{42}\right) a_{5}$

Eigenvalue of $V\left(E_{9}\left(x_{51}, 0, y_{51}, y_{52}\right)\right)$ in $x_{2}$ - direction is

$\xi_{3}=g_{2}(0)-y_{51} p_{2 x_{2}}\left(0, y_{52}\right)-y_{52} q_{2 x_{2}}\left(0, y_{51}\right)$.

Other eigenvalues are given by the roots of

$\lambda^{3}+\left(y_{51} s_{1}^{\prime}+y_{52} s_{2}^{\prime}\right) \lambda^{2}$

$$
\begin{aligned}
& -\left[e_{1}\left(y_{51} s_{1}^{\prime}+y_{52} s_{2}^{\prime}\right)\right. \\
& +\left(y_{51} c_{1} p_{1 x_{1}}\left(p_{1}+y_{52} q_{1 y_{1}}\right)+c_{2} y_{52} q_{1 x_{1}}\left(y_{51} p_{1 y_{2}}+q_{1}\right)\right) \\
& \left.-\left(y_{51} y_{52} s_{1}^{\prime} s_{2}^{\prime}-c_{2} y_{52} q_{1 y_{1}} c_{1} y_{51} p_{1 y_{2}}\right)\right] \lambda \\
& +y_{51} c_{1} p_{1 x_{1}}\left\{\left(p_{1}+y_{52} q_{1 y_{1}}\right) y_{52} s_{2}^{\prime}+c_{2} y_{52} q_{1 y_{1}}\left(y_{51} p_{1 y_{2}}+q_{1}\right)\right\} \\
& +c_{2} y_{52} q_{1 x_{1}}\left\{\left(p_{1}+y_{52} q_{1 y_{1}}\right) c_{1} y_{51} p_{1 y_{2}}+y_{51} s_{1}^{\prime}\left(y_{51} p_{1 y_{2}}+q_{1}\right)\right\}=0
\end{aligned}
$$

where, $e_{1}=g_{1}+x_{1} g_{1}^{\prime}-y_{1} p_{1 x_{1}}-y_{2} q_{1 x_{1}}$.

Above as well as below all functions are evaluated at the equilibrium under consideration. 
Eigenvalue of $V\left(E_{10}\left(0, x_{62}, y_{61}, y_{62}\right)\right)$ in $x_{1}$ - direction is $\xi_{4}=g_{1}(0)-y_{61} p_{1 x_{1}}\left(0, y_{62}\right)-y_{62} q_{1 x_{1}}\left(0, y_{61}\right)$. Other eigenvalues are the roots of $\lambda^{3}+\left(y_{61} s_{1}^{\prime}+y_{62} s_{2}^{\prime}-e_{2}\right) \lambda^{2}$

$$
\begin{aligned}
& -\left[e_{2}\left(y_{61} s_{1}^{\prime}+y_{62} s_{2}^{\prime}\right)\right. \\
& +\left(y_{61} \widetilde{c_{1}} p_{2 x_{2}}\left(p_{2}+y_{62} q_{2 y_{1}}\right)+\widetilde{c_{2}} y_{62} q_{2 x_{2}}\left(y_{61} p_{2 y_{2}}+q_{2}\right)\right)-y_{61} y_{62} s_{1}^{\prime} s_{2}^{\prime} \\
& \left.+\widetilde{c_{2}} y_{61} y_{62} q_{2 y_{1}} \widetilde{c_{1}} p_{2 y_{2}}\right] \lambda \\
& +y_{61} \widetilde{c_{1}} p_{2 x_{2}}\left\{\left(p_{2}+y_{62} q_{2 y_{1}}\right) y_{62} s_{2}^{\prime}+\widetilde{c_{2}} y_{62} q_{2 y_{1}}\left(y_{61} p_{2 y_{2}}+q_{2}\right)\right\} \\
& +\widetilde{c_{2}} y_{62} q_{2 x_{2}}\left\{\left(p_{2}+y_{62} q_{2 y_{1}}\right) \widetilde{c_{1}} y_{61} p_{2 y_{2}}+y_{61} s_{2}^{\prime}\left(y_{61} p_{2 y_{2}}+q_{2}\right)\right\}=0,
\end{aligned}
$$

where, $e_{2}=g_{1}+x_{2} g_{2}^{\prime}-y_{1} p_{2 x_{2}}-y_{2} q_{2 x_{2}}$.

We now obtain conditions of asymptotic stability of interior equilibrium $E^{*}$ :

$A=\left(a_{i j}\right)=\left[\begin{array}{cccc}g_{1}+x_{1} g_{1}^{\prime}-y_{1} p_{1 x_{1}}-y_{2} q_{1 x_{1}} & 0 & -p_{1}-y_{2} q_{1 y_{1}} & -y_{1} p_{1 y_{2}}-q_{1} \\ 0 & g_{2}+x_{2} g_{2}^{\prime}-y_{1} p_{2 x_{2}}-y_{2} q_{2} & -p_{2}-y_{2} q_{2} y_{1} & -y_{1} p_{2 y_{2}}-q_{2} \\ c_{1} y_{1} p_{1 x_{1}} & \widetilde{c_{1}} y_{1} p_{2 x_{2}} & -y_{1} s_{1}^{\prime} & c_{1} y_{1} p_{1 y_{2}}+\widetilde{c_{1}} y_{1} p_{2 y_{2}} \\ c_{2} y_{2} q_{1 x_{1}} & \tilde{c_{2}} y_{2} q_{2} q_{x_{2}} & c_{2} y_{2} q_{1 y_{1}}+\widetilde{c_{2}} y_{2} q_{2 y_{1}} & -y_{2} s_{2}^{\prime}\end{array}\right]$

Computing $\operatorname{det}(A-\lambda I)=0$ we get

$F(\lambda)=\lambda^{4}+\alpha_{1} \lambda^{3}+\alpha_{2} \lambda^{2}+\alpha_{3} \lambda+\alpha_{4}=0$,

where,

$$
\begin{aligned}
& \alpha_{1}=-\sum_{1}^{4} a_{i i}, \\
& \alpha_{2}=m 1-m 2+\left(a_{11}+a_{22}\right)\left(a_{33}+a_{44}\right)+a_{11} a_{22}+\beta_{1}, \\
& \alpha_{3}=-\left(a_{11}+a_{22}\right) m 1-a_{11} a_{22}\left(a_{33}+a_{44}\right)+\gamma_{1}+\gamma_{2}, \\
& \alpha_{4}=m 1 a_{11} a_{22}+\delta_{1}+\delta_{2}+\delta_{3}, \beta_{1}=-\left(a_{13} a_{31}+a_{14} a_{41}\right), \\
& \gamma_{1}=\left(a_{13} a_{22}+m 5\right) a_{31}-a_{41}\left(m 6-a_{14} a_{22}\right), \\
& \delta_{1}=m 6 a_{22} a_{41}-m 5 a_{22} a_{31}, \\
& \gamma_{2}=a_{11} m 2+a_{32} m 3-a_{42} m 4, \delta_{2}=a_{11}\left(a_{42} m 4-a_{32} m 3\right) \\
& \delta_{3}=m 7\left(a_{31} a_{42}-a_{41} a_{32}\right) . \\
& m 1=a_{33} a_{44}-a_{34} a_{43}, m 2=a_{23} a_{32}+a_{24} a_{42}, \\
& m 3=a_{23} a_{44}-a_{24} a_{43}, m 4=a_{23} a_{34}-a_{24} a_{33}, \\
& m 5=a_{13} a_{44}-a_{14} a_{43}, m 6=a_{13} a_{34}-a_{14} a_{33}, \\
& \text { and } m 7=a_{13} a_{24}-a_{14} a_{23 .} .
\end{aligned}
$$

All the entries of matrix $\mathrm{A}$ above are assumed to be computed at $E^{*}\left(x_{1}^{*}, x_{2}^{*}, y_{1}^{*}, y_{2}^{*}\right)$. The result below follows from Hurwitz's theorem [1]: 
Theorem 3: The interior equilibrium $E^{*}\left(x_{1}^{*}, x_{2}^{*}, y_{1}^{*}, y_{2}^{*}\right)$ is asymptotically stable if

$$
\alpha_{i}>0, \quad 1 \leq i \leq 4, \alpha_{1} \alpha_{2} \alpha_{3}-\alpha_{3}^{2}-\alpha_{1}^{2} \alpha_{4}>0 .
$$

\section{Global Stability}

In this section we obtain conditions of global asymptotic stability of four, boundary equlibria that are interior to three dimensional subsystems and the interior equilibrium of (1).

The results of boundary equilibria will be used to obtain conditions for uniform persistence in the next section.

We set $R_{x y z}^{+}=\{(x, y, z) \mid x>0, y>0, z>0\}$, and define $\overline{R_{x y z}^{+}}$to be the closure of $R_{x y z}^{+}$and, so on.

We consider the question of global asymptotically stability of $E_{7}\left(x_{31}, x_{32}, y_{31}, 0\right)$ for the system,

$x_{1}^{\prime}=\alpha x_{1} g_{1}\left(x_{1}\right)-y_{1} p_{1}\left(x_{1}, 0\right)$,

$x_{2}^{\prime}=x_{2} g_{2}\left(x_{2}\right)-y_{1} p_{2}\left(x_{2}, 0\right)$,

$y_{1}^{\prime}=y_{1}\left[-s_{1}\left(y_{1}\right)+c_{1} p_{1}\left(x_{1}, 0\right)+\widetilde{c_{1}} p_{2}\left(x_{2}, 0\right)\right]$

$x_{1}\left(t_{0}\right)=x_{10} \geq 0, x_{2}\left(t_{0}\right)=x_{20} \geq 0, y_{1}\left(t_{0}\right)=y_{10} \geq 0$.

We will find sufficent conditions, such that $E_{7}\left(x_{31}, x_{32}, y_{31}, 0\right)$ is globally asymptotically stable in $R_{x_{1} x_{2} y_{1}}^{+}$.

We define $V\left(x_{1}, x_{2}, y_{1}\right)[4]$ :

$V\left(x_{1}, x_{2}, y_{1}\right)=\sum_{i=1}^{2}\left(x_{i}-x_{3 i}-x_{3 i} \log \frac{x_{i}}{x_{3 i}}\right)+y_{1}-y_{31}-y_{31} \log \frac{y_{1}}{y_{31}}$.

$V$ is positive definite about $E_{7}\left(x_{31}, x_{32}, y_{31}, 0\right)$. Also $V\left(x_{1}, x_{2}, y_{1}\right) \rightarrow+\infty$, as $x_{1}, x_{2}$ and/or $y_{1}$ tend to zero.

Computing $\frac{d V}{d t}$ along the solutions, we get

$$
\begin{aligned}
\frac{d V}{d t} & =\left(x_{1}-x_{31}\right)\left[\alpha g_{1}\left(x_{1}\right)-y_{1} \frac{p_{1}\left(x_{1}, 0\right)}{x_{1}}\right]+\left(x_{2}-x_{32}\right)\left[g_{2}\left(x_{2}\right)-y_{1} \frac{p_{2}\left(x_{2}, 0\right)}{x_{2}}\right] \\
& +\left(y_{1}-y_{31}\right)\left\{-s_{1}\left(y_{1}\right)+c_{1} p_{1}\left(x_{1}, 0\right)+\widetilde{c_{1}} p_{2}\left(x_{2}, 0\right)\right\} \\
= & \sum_{i, j=1}^{3} l_{i j},
\end{aligned}
$$

where, 
$l_{11}=\left(x_{1}-x_{31}\right)\left\{\alpha\left[g_{1}\left(x_{1}\right)-g_{1}\left(x_{31}\right)\right]+y_{1}\left[\frac{p_{1}\left(x_{31}, 0\right)}{x_{31}}-\frac{p_{1}\left(x_{1}, 0\right)}{x_{1}}\right]\right\}$,

$l_{12}=0, l_{13}=-\frac{\left(x_{1}-x_{31}\right)}{x_{31}}\left(y_{1}-y_{31}\right) p_{1}\left(x_{31}, 0\right)$,

$l_{21}=0, l_{22}=\left(x_{2}-x_{32}\right)\left\{g_{2}\left(x_{2}\right)-g_{2}\left(x_{32}\right)+y_{1}\left[\frac{p_{2}\left(x_{32}, 0\right)}{x_{32}}-\frac{p_{2}\left(x_{2}, 0\right)}{x_{2}}\right]\right\}$,

$l_{23}=-\frac{1}{x_{32}}\left(x_{2}-x_{32}\right)\left(y_{1}-y_{31}\right) p_{2}\left(x_{32}, 0\right), l_{31}=c_{1}\left(y_{1}-y_{31}\right)\left[p_{1}\left(x_{1}, 0\right)-\right.$ $\left.p_{1}\left(x_{31}, 0\right)\right]$,

$l_{32}=\widetilde{c_{1}}\left(y_{1}-y_{31}\right)\left[p_{2}\left(x_{2}, 0\right)-p_{2}\left(x_{32}, 0\right)\right], l_{33}=-\left(y_{1}-y_{31}\right)\left[s_{1}\left(y_{1}\right)-s_{1}\left(y_{31}\right)\right]$.

We set

$l_{11}=\left(x_{1}-x_{31}\right)^{2} m_{11}\left(x_{1}, y_{1}\right), m_{12}=0$,

$l_{13}+l_{31}=2\left(x_{1}-x_{31}\right)\left(y_{1}-y_{31}\right) m_{13}\left(x_{1}\right)$,

$l_{22}=\left(x_{2}-x_{32}\right)^{2} m_{22}\left(x_{2}\right)$,

$l_{23}+l_{32}=2\left(x_{2}-x_{32}\right)\left(y_{1}-y_{31}\right) m_{23}\left(x_{2}\right)$,

$l_{33}=\left(y_{1}-y_{31}\right)^{2} m_{33}\left(y_{1}\right)$,

and

$m_{i j}=m_{j i}, \quad i>j$.

Thus

$\frac{d V}{d t}=x^{T} M x$

where, $\mathbf{M}=\left(m_{i j}\right), x=\left(\begin{array}{l}x_{1}-x_{31} \\ x_{2}-x_{32} \\ y_{1}-y_{31}\end{array}\right)$.

Also from Theorem 2 we have that $\mathcal{A}_{1}=$ $\left\{\left(x_{1}, x_{2}, y_{1}\right) \mid 0 \leq x_{1} \leq K_{1}, 0 \leq x_{2} \leq K_{2}, c_{1} x_{1}+\widetilde{c_{1}} x_{2}+y_{1} \leq \frac{c_{1} G_{1}+\widetilde{c_{1}} G_{2}}{s_{1}(0)}\right\}$ is an attracting set for the subsystem in $R_{x_{1} x_{2} y_{1}}^{+}$.

Theorem 4: Let the symmetric matrix $M$ be negative definite in $\mathcal{A}_{1}$. Then $E_{7}\left(x_{31}, x_{32}, y_{31}, 0\right)$ is globally asymptotically stable in $R_{x_{1} x_{2} y_{1}}^{+}$.

Proof : The solutions are bounded and the largest invariant set of $\left\{x \in R_{x_{1} x_{2} y_{1}}^{+} \mid \dot{V}=0\right\}=\left\{E_{7}\right\}$. Hence $E_{7}$ is globally asymptotically stable in $R_{x_{1} x_{2} y_{1}}^{+}[11]$.

Next we consider the question of global asymptotically stability of $E_{8}\left(x_{41}, x_{42}, 0, y_{42}\right)$ 
for the system

$x_{1}^{\prime}=\alpha x_{1} g_{1}\left(x_{1}\right)-y_{2} q_{1}\left(x_{1}, 0\right)$,

$x_{2}^{\prime}=x_{2} g_{2}\left(x_{2}\right)-y_{2} q_{2}\left(x_{2}, 0\right)$,

$y_{2}^{\prime}=y_{2}\left[-s_{2}\left(y_{2}\right)+c_{2} q_{1}\left(x_{1}, 0\right)+\widetilde{c_{2}} q_{2}\left(x_{2}, 0\right)\right]$

$x_{1}\left(t_{0}\right)=x_{10} \geq 0, x_{2}\left(t_{0}\right)=x_{20} \geq 0, y_{2}\left(t_{0}\right)=y_{20} \geq 0$.

We define a Lyapunov function $\mathrm{V}\left(\left(x_{1}, x_{2}, y_{2}\right)\right.$,

$V\left(x_{1}, x_{2}, y_{2}\right)=\sum_{i=1}^{2}\left(x_{i}-x_{4 i}-x_{4 i} \log \frac{x_{i}}{x_{4 i}}\right)+y_{2}-y_{42}-y_{42} \log \frac{y_{2}}{y_{42}}$.

$V$ is positive definite about $E_{8}\left(x_{41}, x_{42}, 0, y_{42}\right)$. Also $V\left(x_{1}, x_{2}, y_{2}\right) \rightarrow+\infty$, as $x_{i}$ and/or $y_{2}$ tend to zero, $i=1,2$. Computing time derivative of $\mathrm{V}$ along the solutions, we get,

$$
\begin{gathered}
\frac{d V}{d t}=\left(x_{1}-x_{41}\right)\left\{\alpha g_{1}\left(x_{1}\right)-\frac{y_{2}}{x_{1}} q_{1}\left(x_{1}, 0\right)\right\}+\left(x_{2}-x_{42}\right)\left\{g_{2}\left(x_{2}\right)-\frac{y_{2}}{x_{2}} q_{2}\left(x_{2}, 0\right)\right\} \\
+\left(y_{2}-y_{42}\right)\left\{-s_{2}\left(y_{2}\right)+c_{2} q_{1}\left(x_{1}, 0\right)+\widetilde{c_{2}} q_{2}\left(x_{2}, 0\right)\right\}
\end{gathered}
$$

$=\sum_{i, j=1}^{3} l_{i j}$

$$
\begin{aligned}
& l_{11}=\left(x_{1}-x_{41}\right)\left\{\alpha\left[g_{1}\left(x_{1}\right)-g_{1}\left(x_{41}\right)\right]+y_{2}\left[\frac{q_{1}\left(x_{41}, 0\right)}{x_{41}}-\frac{q_{1}\left(x_{1}, 0\right)}{x_{1}}\right]\right\}, \\
& l_{12}=0, l_{13}=-\frac{\left(x_{1}-x_{41}\right)}{x_{41}}\left(y_{2}-y_{42}\right) q_{1}\left(x_{41}, 0\right), \\
& l_{21}=0, l_{22}=\left(x_{2}-x_{42}\right)\left\{g_{2}\left(x_{2}\right)-g_{2}\left(x_{42}\right)+y_{2}\left[\frac{q_{2}\left(x_{42}, 0\right)}{x_{42}}-\frac{q_{2}\left(x_{2}, 0\right)}{x_{2}}\right]\right\}, \\
& l_{23}=-\frac{1}{x_{42}}\left(x_{2}-x_{42}\right)\left(y_{2}-y_{42}\right) q_{2}\left(x_{42}, 0\right), \\
& l_{31}=c_{2}\left(y_{2}-y_{42}\right)\left[q_{1}\left(x_{1}, 0\right)-q_{1}\left(x_{41}, 0\right)\right], l_{32} \\
& \quad=\widetilde{c_{2}}\left(y_{2}-y_{42}\right)\left[q_{2}\left(x_{2}, 0\right)-q_{2}\left(x_{42}, 0\right)\right], \\
& l_{33}=-\left(y_{2}-y_{42}\right)\left[s_{2}\left(y_{2}\right)-s_{2}\left(y_{42}\right)\right] .
\end{aligned}
$$

Next, we define $n_{i j}{ }^{\prime} s$, such that

$l_{11}=\left(x_{1}-x_{41}\right)^{2} n_{11}\left(x_{1}, y_{2}\right), \quad n_{12}=0, l_{13}+l_{31}=2\left(x_{1}-x_{41}\right)\left(y_{2}-\right.$

$\left.y_{42}\right) n_{13}\left(x_{1}\right)$,

$l_{22}=\left(x_{2}-x_{42}\right)^{2} n_{22}\left(x_{2}, y_{2}\right), l_{23}+l_{32}=2\left(x_{2}-x_{42}\right)\left(y_{2}-y_{42}\right) n_{23}\left(x_{2}\right)$,

$l_{33}=\left(y_{2}-y_{42}\right)^{2} n_{33}\left(y_{2}\right)$,

and 
$n_{i j}=n_{j i}, \quad i>j$.

Thus

$\frac{d V}{d t}=x^{T} N x$

$N=\left(n_{i j}\right), \quad x=\left(\begin{array}{l}x_{1}-x_{41} \\ x_{2}-x_{42} \\ y_{2}-y_{42}\end{array}\right)$

Also from Theorem 2 we have: $\mathcal{A}_{2}=$ $\left\{\left(x_{1}, x_{2}, y_{2}\right) \mid 0 \leq x_{1} \leq K_{1}, 0 \leq x_{2} \leq K_{2}, c_{2} x_{2}+\widetilde{c_{2}} x_{2}+y_{2} \leq \frac{c_{2} L_{1}+\widetilde{c_{2}} L_{2}}{s_{2}(0)}\right\}$ is an attracting set for the subsystem in $R_{x_{1} x_{2} y_{2}}^{+}$. Proceeding as in Theorem 4, we get:

Theorem 5: Let the symmetric matrix $\mathrm{N}$ be negative definite in $\mathcal{A}_{2}$. Then $E_{8}\left(x_{41}, x_{42}, 0, y_{42}\right)$ is globally asymptotically stable in $R_{x_{1} x_{2} y_{2}}^{+}$.

Next we consider global stability of $E_{9}$ for the submodel in $R_{x_{1} y_{1} y_{2}}^{+}$: we define a Lyapunov function $V\left(x_{1}, y_{1}, y_{2}\right)$ :

$V\left(x_{1}, y_{1}, y_{2}\right)=\left(x_{1}-x_{51}-x_{51} \log \frac{x_{1}}{x_{51}}\right)+\sum_{i=1}^{2}\left(y_{i}-y_{5 i}-y_{5 i} \log \frac{y_{i}}{y_{5 i}}\right)$.

$V$ is positive definite about $E_{9}\left(x_{51}, 0, y_{51}, y_{52}\right)$. Also as $x_{i}$ and/or $y_{i} \rightarrow 0, V \rightarrow+\infty$, $i=1,2$.

Computing the time derivative of $\mathrm{V}$ along the solutions of we get,

$$
\begin{aligned}
& \frac{d V}{d t}=\left(x_{1}-x_{51}\right)\left\{\alpha g_{1}\left(x_{1}\right)-y_{1} \frac{p_{1}\left(x_{1}, y_{2}\right)}{x_{1}}-y_{2} \frac{q_{1}\left(x_{1}, y_{1}\right)}{x_{1}}\right\} \\
& +\left(y_{1}-y_{52}\right)\left\{-s_{1}\left(y_{1}\right)+c_{1} p_{1}\left(x_{1}, y_{2}\right)\right\} \\
& +\left(y_{2}-y_{52}\right)\left\{-s_{2}\left(y_{2}\right)+c_{2} q_{1}\left(x_{1}, y_{1}\right)\right\}
\end{aligned}
$$

where,

$$
\begin{aligned}
& l_{11}=\left(x_{1}-x_{51}\right)\left\{\alpha\left[g_{1}\left(x_{1}\right)-g_{1}\left(x_{51}\right)\right]+y_{1}\left(\frac{p_{1}\left(x_{51}, y_{52}\right)}{x_{51}}-\frac{p_{1}\left(x_{1}, y_{52}\right)}{x_{1}}\right)+\right. \\
& \left.y_{2}\left(\frac{q_{1}\left(x_{51}, y_{51}\right)}{x_{51}}-\frac{q_{1}\left(x_{51}, y_{51}\right)}{x_{1}}\right)\right\}, \\
& l_{12}=-\frac{\left(x_{1}-x_{51}\right)}{x_{51}}\left\{\left(y_{1}-y_{51}\right) p_{1}\left(x_{51}, y_{52}\right)+\frac{y_{2}}{x_{1}}\left[q_{1}\left(x_{1}, y_{1}\right)-q_{1}\left(x_{1}, y_{51}\right)\right]\right\}, \\
& l_{13}=-\left(x_{1}-x_{51}\right)\left\{\frac{y_{1}}{x_{51}}\left[p_{1}\left(x_{1}, y_{2}\right)-p_{1}\left(x_{1}, y_{52}\right)\right]+\frac{\left(y_{2}-y_{52}\right)}{x_{51}} q_{1}\left(x_{51}, y_{51}\right)\right\}, \\
& l_{21}=c_{1}\left(y_{1}-y_{51}\right)\left\{p_{1}\left(x_{1}, y_{2}\right)-p_{1}\left(x_{51}, y_{2}\right)\right\},
\end{aligned}
$$




$$
\begin{aligned}
& l_{22}=-\left(y_{1}-y_{51}\right)\left[s_{1}\left(y_{1}\right)-s_{1}\left(y_{51}\right)\right], \\
& l_{23}=c_{1}\left(y_{1}-y_{51}\right)\left[p_{1}\left(x_{51}, y_{2}\right)-p_{1}\left(x_{51}, y_{52}\right)\right], \\
& l_{31}=\left(y_{2}-y_{52}\right) c_{2}\left\{q_{1}\left(x_{1}, y_{1}\right)-q_{1}\left(x_{51}, y_{1}\right)\right\}, \\
& l_{32}=c_{2}\left(y_{2}-y_{52}\right)\left[-q_{1}\left(x_{51}, y_{51}\right)+q_{1}\left(x_{51}, y_{1}\right)\right], \\
& l_{33}=-\left(y_{2}-y_{52}\right)\left[s_{2}\left(y_{2}\right)-s_{2}\left(y_{52}\right)\right] .
\end{aligned}
$$

We set

$$
\begin{aligned}
& l_{11}=\left(x_{1}-x_{1}^{*}\right)^{2} r_{11}\left(x_{1}, y_{1}, y_{2}\right), l_{12}+l_{21}=2\left(x_{1}-x_{51}\right)\left(y_{1}-y_{51}\right) r_{12}\left(x_{1}, y_{1}, y_{2}\right), \\
& l_{13}+l_{31}=2\left(x_{1}-x_{51}\right)\left(y_{2}-y_{52}\right) r_{13}\left(x_{1}, y_{1}, y_{2}\right), l_{22}=\left(y_{1}-y_{51}\right)^{2} r_{22}\left(y_{1}\right), \\
& l_{23}+l_{32}=2\left(y_{1}-y_{51}\right) r_{23}\left(y_{2}\right)\left(y_{2}-y_{52}\right), l_{33}=\left(y_{2}-y_{52}\right)^{2} r_{33}\left(y_{2}\right) .
\end{aligned}
$$

\section{Define}

$r_{i j}=r_{j i}, i>j, R=\left(r_{i j}\right)$,

So, $\dot{V}=x^{T} R x$,

Also from Theorem 2, $\mathcal{A}_{3}=$ $\left\{\left(x_{1}, y_{1}, y_{2}\right) \mid 0 \leq x_{1} \leq K_{1}, 0 \leq c_{1} x_{1}+y_{1} \leq \frac{c_{1} G_{1}}{s_{1}(0)}, c_{2} x_{1}+y_{2} \leq \frac{c_{2} L_{1}}{s_{2}(0)}\right\}$ is an attracting set for the subsystem in $R_{x_{1} y_{1} y_{2}}^{+}$.

where, $\mathrm{R}=\left(r_{i j}\right)$ and $x=\left(\begin{array}{l}x_{1}-x_{51} \\ y_{1}-y_{51} \\ y_{2}-y_{52}\end{array}\right)$.

We obtain the following result:

Theorem 6: Whenever the symmetric matrix $\mathrm{R}$ is negative definite on $\mathcal{A}_{3}$, the equilibrium $E_{9}\left(x_{51}, 0, y_{51}, y_{52}\right)$ is globally asymptotically stable in $R_{x_{1} y_{1} y_{2}}^{+}$.

In order to obtain global asymptotic criteria for the equilibrium $E_{10}\left(0, x_{62}, y_{61}, y_{62}\right)$ in $R_{x_{2} y_{1} y_{2}}^{+}$, We define a Lyapunov function $V\left(x_{2}, y_{1}, y_{2}\right)$,

$V\left(x_{2}, y_{1}, y_{2}\right)=\left(x_{2}-x_{62}-x_{62} \log \frac{x_{2}}{x_{62}}\right)+\sum_{i=1}^{2}\left(y_{i}-y_{6 i}-y_{6 i} \log \frac{y_{i}}{y_{6 i}}\right)$.

$V$ is positive definite about $E_{10}\left(0, x_{62}, y_{61}, y_{62}\right)$. Also $V \rightarrow+\infty$ as $x_{2}$ or $y_{i}$ tend to $0+, i=1,2$.

Computing the time derivative of $\mathrm{V}$ along the solutions, we get 


$$
\begin{gathered}
\frac{d V}{d t}=\left(x_{2}-x_{62}\right)\left\{g_{2}\left(x_{2}\right)-y_{1} \frac{p_{2}\left(x_{2}, y_{2}\right)}{x_{2}}-y_{2} \frac{q_{2}\left(x_{2}, y_{1}\right)}{x_{2}}\right\} \\
+\left(y_{1}-y_{62}\right)\left\{-s_{1}\left(y_{1}\right)+c_{1} p_{1}\left(x_{2}, y_{2}\right)\right\} \\
+\left(y_{2}-y_{62}\right)\left\{-s_{2}\left(y_{2}\right)+c_{2} q_{1}\left(x_{2}, y_{1}\right)\right\} \\
=\sum_{i, j=1}^{3} l_{i j},
\end{gathered}
$$

where,

$$
\begin{aligned}
& l_{11}=\left(x_{2}-x_{62}\right)\left\{g_{2}\left(x_{2}\right)-g_{2}\left(x_{62}\right)+y_{1}\left(\frac{p_{2}\left(x_{62}, y_{62}\right)}{x_{62}}-\frac{p_{2}\left(x_{2}, y_{62}\right)}{x_{2}}\right)+y_{2}\left(\frac{q_{2}\left(x_{62}, y_{61}\right)}{x_{62}}-\right.\right. \\
& \left.\left.\frac{q_{2}\left(x_{2}, y_{61}\right)}{x_{2}}\right)\right\}, \\
& l_{12}=-\left(x_{2}-x_{62}\right)\left\{\frac{1}{x_{62}}\left(y_{1}-y_{61}\right) p_{2}\left(x_{62}, y_{62}\right)+\frac{y_{2}}{x_{2}}\left[q_{2}\left(x_{2}, y_{1}\right)-q_{2}\left(x_{2}, y_{61}\right)\right]\right\}, \\
& l_{13}=-\left(x_{2}-x_{62}\right)\left\{\frac{y_{1}}{x_{62}}\left[p_{2}\left(x_{2}, y_{2}\right)-p_{2}\left(x_{2}, y_{62}\right)\right]+\frac{\left(y_{2}-y_{62}\right)}{x_{62}} q_{2}\left(x_{62}, y_{61}\right)\right\}, \\
& l_{21}=\widetilde{c_{1}}\left(y_{1}-y_{61}\right)\left\{p_{2}\left(x_{2}, y_{2}\right)-p_{2}\left(x_{62}, y_{2}\right)\right\}, l_{22}=\left(y_{1}-y_{61}\right)\left[s_{1}\left(y_{1}\right)-s_{1}\left(y_{61}\right)\right], \\
& l_{23}=\left(y_{1}-y_{61}\right) \widetilde{c_{1}}\left[p_{2}\left(x_{62}, y_{2}\right)-p_{2}\left(x_{62}, y_{62}\right)\right], l_{31}=\left(y_{2}-y_{62}\right) \widetilde{c_{2}}\left\{q_{2}\left(x_{2}, y_{1}\right)-\right. \\
& \left.q_{2}\left(x_{62}, y_{1}\right)\right\}, \\
& l_{32}=\widetilde{c_{2}}\left(y_{2}-y_{62}\right)\left[-q_{2}\left(x_{62}, y_{61}\right)+q_{2}\left(x_{62}, y_{1}\right)\right], l_{33}=\left(y_{2}-y_{62}\right)\left[s_{2}\left(y_{2}\right)-\right. \\
& \left.s_{2}\left(y_{62}\right)\right] .
\end{aligned}
$$

We set

$$
\begin{aligned}
& l_{11}=\left(x_{2}-x_{62}\right)^{2} t_{11}\left(x_{2}, y_{1}, y_{2}\right), l_{12}+l_{21}=2\left(x_{2}-x_{62}\right)\left(y_{1}-y_{61}\right) t_{12}\left(x_{2}, y_{1}, y_{2}\right), \\
& l_{13}+l_{31}=2\left(x_{2}-x_{62}\right)\left(y_{2}-y_{62}\right) t_{13}\left(x_{2}, y_{1}, y_{2}\right), l_{22}=\left(y_{1}-y_{61}\right)^{2} t_{22}\left(y_{1}\right), \\
& l_{23}+l_{32}=2\left(y_{1}-y_{61}\right) t_{23}\left(y_{2}\right)\left(y_{2}-y_{62}\right), l_{33}=\left(y_{2}-y_{62}\right)^{2} t_{33}\left(y_{2}\right),
\end{aligned}
$$

Define

$t_{i j}=t_{j i}, i>j, T=\left(t_{i j}\right)$.

So

$\dot{V}=x^{T} T x$,

Where, $=\left(\begin{array}{l}x_{2}-x_{62} \\ y_{1}-y_{61} \\ y_{2}-y_{62}\end{array}\right)$.

Also from Theorem 2, $\mathcal{A}_{4}=$

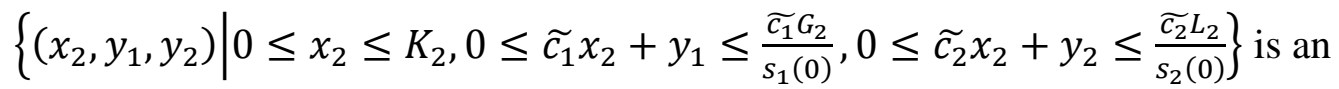
attracting set for the subsystem in $R_{x_{2}}^{+} y_{1} y_{2}$. Thus we have: 
Theorem 7: Whenever the symmetric matrix $\mathrm{T}$ is negative definite in the region $\mathcal{A}_{4}$, the equilibrium $E_{10}\left(0, x_{62}, y_{51}, y_{52}\right)$ is globally asymptotically stable in $R_{x_{2 y_{1} y_{2}}^{+}}$. Lastly we derive conditions for global stability of interior equilibrium $E^{*}$ Define a Lyapunov function $V\left(x_{1}, x_{2}, y_{1}, y_{2}\right)$.

$$
V\left(x_{1}, x_{2}, y_{1}, y_{2}\right)=\sum_{i=1}^{2}\left(x_{i}-x_{i}^{*}-x_{i}^{*} \log \frac{x_{i}}{x_{i}^{*}}\right)+\sum_{i=1}^{2}\left(y_{i}-y_{i}^{*}-y_{i}^{*} \log \frac{y_{i}}{y_{i}^{*}}\right)
$$

$V$ is positive definite about $E^{*}\left(x_{1}^{*}, x_{2}^{*}, y_{1}^{*}, y_{2}^{*}\right)$.Also as $x_{i}$ and/or $y_{i} \rightarrow 0, V \rightarrow+\infty, i=$ 1,2 .

Computing the time derivate of $\mathrm{V}$ along the solutions of (1) we get,

$$
\begin{aligned}
\frac{d V}{d t}=\left(x_{1}-x_{1}^{*}\right) & \left\{\alpha g_{1}\left(x_{1}\right)-y_{1} \frac{p_{1}\left(x_{1}, y_{2}\right)}{x_{1}}-y_{2} \frac{q_{1}\left(x_{1}, y_{1}\right)}{x_{1}}\right\} \\
& +\left(x_{2}-x_{2}^{*}\right)\left\{g_{2}\left(x_{2}\right)-y_{1} \frac{p_{2}\left(x_{2}, y_{2}\right)}{x_{2}}-y_{2} \frac{q_{2}\left(x_{2}, y_{1}\right)}{x_{2}}\right\} \\
& +\left(y_{1}-y_{1}^{*}\right)\left\{-s_{1}\left(y_{1}\right)+c_{1} p_{1}\left(x_{1}, y_{2}\right)+\widetilde{c_{1}} p_{2}\left(x_{2}, y_{2}\right)\right\} \\
& +\left(y_{2}-y_{2}^{*}\right)\left\{-s_{2}\left(y_{2}\right)+c_{2} q_{1}\left(x_{1}, y_{1}\right)+\widetilde{c_{2}} q_{2}\left(x_{2}, y_{1}\right)\right\}
\end{aligned}
$$

$=\sum_{i, j=1}^{4} l_{i j}$,

where,

$$
\begin{aligned}
& l_{11}=\left(x_{1}-x_{1}^{*}\right)\left\{\alpha\left[g_{1}\left(x_{1}\right)-g_{1}\left(x_{1}^{*}\right)\right]+y_{1}\left(\frac{p_{1}\left(x_{1}^{*}, y_{2}^{*}\right)}{x_{1}^{*}}-\frac{p_{1}\left(x_{1}, y_{2}^{*}\right)}{x_{1}}\right)+y_{2}\left(\frac{q_{1}\left(x_{1}^{*}, y_{1}^{*}\right)}{x_{1}^{*}}-\frac{q_{1}\left(x_{1}, y_{1}^{*}\right)}{x_{1}}\right)\right\}, \\
& l_{12}=0 \\
& l_{13}=-\left(x_{1}-x_{1}^{*}\right)\left\{\left(y_{1}-y_{1}^{*}\right) \frac{p_{1}\left(x_{1}^{*}, y_{2}^{*}\right)}{x_{1}^{*}}+\frac{y_{2}}{x_{1}}\left[q_{1}\left(x_{1}, y_{1}\right)-q_{1}\left(x_{1}, y_{1}^{*}\right)\right]\right\}, \\
& l_{14}=-\left(x_{1}-x_{1}^{*}\right)\left\{\frac{y_{1}}{x_{1}}\left[p_{1}\left(x_{1}, y_{2}\right)-p_{1}\left(x_{1}, y_{2}^{*}\right)\right]+\frac{\left(y_{2}-y_{2}^{*}\right)}{x_{1}^{*}} q_{1}\left(x_{1}^{*}, y_{1}^{*}\right)\right\}, \\
& l_{21}=0 \\
& l_{22}=\left(x_{2}-x_{2}^{*}\right)\left\{g_{2}\left(x_{2}\right)-g_{2}\left(x_{2}^{*}\right)+y_{1}\left[\frac{p_{2}\left(x_{2}^{*}, y_{2}^{*}\right)}{x_{2}^{*}}-\frac{p_{2}\left(x_{2}, y_{2}^{*}\right)}{x_{2}}\right]+y_{2}\left[\frac{q_{2}\left(x_{2}^{*}, y_{1}^{*}\right)}{x_{2}^{*}}-\frac{q_{2}\left(x_{2}, y_{1}^{*}\right)}{x_{2}}\right]\right\}, \\
& l_{23}=-\left(x_{2}-x_{2}^{*}\right)\left\{\frac{y_{2}}{x_{2}}\left[q_{2}\left(x_{2}, y_{1}\right)-q_{2}\left(x_{2}, y_{1}^{*}\right)\right]+\frac{1}{x_{2}^{*}}\left[\left(y_{1}-y_{1}^{*}\right) p_{2}\left(x_{2}^{*}, y_{2}^{*}\right)\right]\right\}, \\
& l_{24}=-\left(x_{2}-x_{2}^{*}\right)\left\{\frac{y_{1}}{x_{2}}\left[p_{2}\left(x_{2}, y_{2}\right)-p_{2}\left(x_{2}, y_{2}^{*}\right)\right]+\frac{q_{2}\left(x_{2}^{*}, y_{1}^{*}\right)}{x_{2}^{*}}\left(y_{2}-y_{2}^{*}\right)\right\}, \\
& l_{31}=c_{1}\left(y_{1}-y_{1}^{*}\right)\left\{p_{1}\left(x_{1}, y_{2}\right)-p_{1}\left(x_{1}^{*}, y_{2}\right)\right\}, \\
& l_{32}=\widetilde{c_{1}}\left(y_{1}-y_{1}^{*}\right)\left\{p_{2}\left(x_{2}, y_{2}\right)-p_{2}\left(x_{2}^{*}, y_{2}\right)\right\},
\end{aligned}
$$


$l_{33}=-\left(y_{1}-y_{1}^{*}\right)\left[s_{1}\left(y_{1}\right)-s_{1}\left(y_{1}^{*}\right)\right]$,

$l_{34}=\left(y_{1}-y_{1}^{*}\right)\left\{\left[p_{1}\left(x_{1}^{*}, y_{2}\right)-p_{1}\left(x_{1}^{*}, y_{2}^{*}\right)\right] c_{1}+\left[p_{2}\left(x_{2}^{*}, y_{2}\right)-p_{2}\left(x_{2}^{*}, y_{2}^{*}\right)\right] \widetilde{c_{1}}\right\}$,

$l_{41}=\left(y_{2}-y_{2}^{*}\right) c_{2}\left\{q_{1}\left(x_{1}, y_{1}\right)-q_{1}\left(x_{1}^{*}, y_{1}\right)\right\}$,

$l_{42}=\left(y_{2}-y_{2}^{*}\right) \widetilde{c_{2}}\left\{q_{2}\left(x_{2}, y_{1}\right)-q_{2}\left(x_{2}^{*}, y_{1}\right)\right\}$,

$l_{43}=\left(y_{2}-y_{2}^{*}\right)\left\{c_{2}\left[-q_{1}\left(x_{1}^{*}, y_{1}^{*}\right)+q_{1}\left(x_{1}^{*}, y_{1}\right)\right]+\widetilde{c_{2}}\left[q_{2}\left(x_{2}^{*}, y_{1}\right)-q_{2}\left(x_{2}^{*}, y_{1}^{*}\right)\right]\right\}$,

$l_{44}=-\left(y_{2}-y_{2}^{*}\right)\left[s_{2}\left(y_{2}\right)-s_{2}\left(y_{2}^{*}\right)\right]$.

We set

$l_{11}=\left(x_{1}-x_{1}^{*}\right)^{2} u_{11}\left(x_{1}, y_{1}, y_{2}\right)$,

$l_{12}=0, l_{13}+l_{31}=2\left(x_{1}-x_{1}^{*}\right)\left(y_{1}-y_{1}^{*}\right) u_{13}\left(x_{1}, y_{1}, y_{2}\right)$,

$l_{14}+l_{41}=2\left(x_{1}-x_{1}^{*}\right)\left(y_{2}-y_{2}^{*}\right) u_{14}\left(x_{1}, y_{1}, y_{2}\right), l_{21}=0$,

$l_{22}=\left(x_{2}-x_{2}^{*}\right)^{2} u_{22}\left(x_{2}, y_{1}, y_{2}\right), l_{23}+l_{32}=2\left(x_{2}-x_{2}^{*}\right)\left(y_{1}-y_{1}^{*}\right) u_{23}\left(x_{2}, y_{1}, y_{2}\right)$,

$l_{24}+l_{42}=2\left(x_{2}-x_{2}^{*}\right)\left(y_{2}-y_{2}^{*}\right) u_{24}\left(x_{2}, y_{1}, y_{2}\right), l_{33}=\left(y_{1}-y_{1}^{*}\right)^{2} u_{33}\left(y_{1}\right)$,

$l_{34}+l_{43}=2\left(y_{1}-y_{1}^{*}\right) u_{34}\left(y_{2}\right)\left(y_{2}-y_{2}^{*}\right), l_{44}=\left(y_{2}-y_{2}^{*}\right)^{2} u_{44}\left(y_{2}\right)$,

Define

$u_{i j}=u_{j i}, i>j$.

So, $\quad \dot{V}=x^{T} U x$,

where, $U=\left(u_{i j}\right)$ and $x=\left(\begin{array}{l}x_{1}-x_{1}^{*} \\ x_{2}-x_{2}^{*} \\ y_{1}-y_{1}^{*} \\ y_{2}-y_{2}^{*}\end{array}\right)$.

Theorem 8: When matrix $\mathrm{U}$ is negative definite on $\mathcal{A}$, the equilibrium $E^{*}\left(x_{1}^{*}, x_{2}^{*}, y_{1}^{*}, y_{2}^{*}\right)$ is globally asymptotically stable in $R_{x_{1} x_{2} y_{1} y_{2}}^{+}$.

Proof : The solutions are bounded and the largest invariant set of $\left\{x \in R_{x_{1} x_{2} y_{1} y_{2}}^{+} \mid \dot{V}=0\right\}=\left\{E^{*}\right\}$. Hence the result follows from [11].

\section{Uniform Persistence}

We study the question of uniform persistence [2] of system (1) in this section.

Define

$$
\begin{aligned}
& P(x)=x_{1}^{\gamma_{1}} x_{2}^{\gamma_{2}} y_{1}^{\delta} y_{2}^{\mu}\left(x_{1}+x_{2}\right)^{\epsilon} \\
& \ln P=\gamma_{1} \ln x_{1}+\gamma_{2} \ln x_{2}+\delta \ln y_{1}+\mu \ln y_{2}+\epsilon \ln \left(x_{1}+x_{2}\right)
\end{aligned}
$$




$$
\begin{aligned}
\psi=\frac{d \ln P}{d t}=\frac{1}{P} & \dot{P} \\
=\gamma_{1}\left(g_{1}\left(x_{1}\right)-\right. & \left.y_{1} \frac{p_{1}\left(x_{1}, y_{2}\right)}{x_{1}}-y_{2} \frac{q_{1}\left(x_{1}, y_{1}\right)}{x_{1}}\right) \\
& +\gamma_{2}\left(g_{2}\left(x_{2}\right)-y_{1} \frac{p_{2}\left(x_{2}, y_{2}\right)}{x_{2}}-y_{2} \frac{q_{2}\left(x_{2}, y_{1}\right)}{x_{2}}\right) \\
& +\delta\left(-s_{1}\left(y_{1}\right)+c_{1} p_{1}\left(x_{1}, y_{2}\right)+\widetilde{c_{1}} p_{2}\left(x_{2}, y_{2}\right)\right) \\
& +\mu\left(-s_{2}\left(y_{2}\right)+c_{2} q_{1}\left(x_{1}, y_{1}\right)+\widetilde{c_{2}} q_{2}\left(x_{2}, y_{1}\right)\right) \\
& +\frac{\epsilon}{x_{1}+x_{2}}\left[\left(x_{1} g_{1}-y_{1} p_{1}-y_{2} q_{1}\right)+x_{2} g_{2}-y_{1} p_{2}-y_{2} q_{2}\right]
\end{aligned}
$$

It is sufficient to show ([6]), that there exist positive constants $\gamma_{1}, \gamma_{2}, \delta, \mu$ and $\epsilon$ such that $\psi>0$ at all boundary equilibria. That is we require,

$$
\begin{aligned}
& \gamma_{1} g_{1}(0)+\gamma_{2} g_{2}(0)-s_{1}(0) \delta-s_{2}(0) \mu+\epsilon \min \left(g_{1}(0), g_{2}(0)\right)>0 \\
& \gamma_{2} g_{2}(0)+\delta\left(-s_{1}(0)+c_{1} p_{1}\left(K_{1}, 0\right)\right)+\mu\left(-s_{2}(0)+c_{2} q_{1}\left(K_{1}, 0\right)\right)>0 \\
& g_{1}(0) \gamma_{1}+\delta\left(-s_{1}(0)+\widetilde{c_{1}} p_{2}\left(K_{2}, 0\right)\right)+\mu\left(-s_{2}(0)+\widetilde{c_{2}} q_{2}\left(K_{2}, 0\right)\right)>0 \\
& \gamma_{2}\left(g_{2}(0)-\overline{y_{1}} p_{2_{x_{2}}}(0,0)\right)+\mu\left(-s_{2}(0)+c_{2} q_{1}\left(\overline{x_{1}}, \overline{y_{1}}\right)\right)>0 \\
& \gamma_{1}\left(g_{1}(0)-\overline{y_{11}} p_{1_{x_{1}}}(0,0)\right)+\mu\left(-s_{2}(0)+\widetilde{c_{2}} q_{2}\left(\overline{x_{2}}, \overline{y_{11}}\right)\right)>0 \\
& \gamma_{2}\left(g_{2}(0)-\widetilde{y_{2}} q_{2_{x_{2}}}(0,0)\right)+\delta\left(-s_{1}(0)+c_{1} p_{1}\left(\widetilde{x_{1}}, \widetilde{y_{2}}\right)\right)>0 \\
& \gamma_{1}\left(g_{1}(0)-\widetilde{y_{22}} q_{1_{x_{1}}}(0,0)\right)+\delta\left(-s_{1}(0)+\widetilde{c_{1}} p_{2}\left(\widetilde{x_{2}}, \widetilde{y_{22}}\right)\right)>0 \\
& \mu\left(-s_{2}(0)+c_{2} q_{1}\left(x_{31}, y_{31}\right)+\widetilde{c_{2}} q_{2}\left(x_{32}, y_{31}\right)\right)>0 \\
& \delta\left(-s_{1}(0)+c_{1} p_{1}\left(x_{41}, y_{42}\right)+\widetilde{c_{1}} p_{2}\left(x_{42}, y_{42}\right)\right)>0 \\
& \gamma_{2}\left(g_{2}(0)-y_{51} p_{2_{x_{2}}}\left(0, y_{52}\right)-y_{52} q_{2_{x_{2}}}\left(0, y_{51}\right)\right)>0 \\
& \gamma_{1}\left(g_{1}(0)-y_{61} p_{1_{x_{1}}}\left(0, y_{62}\right)-y_{62} q_{x_{1}}\left(0, y_{61}\right)\right)>0
\end{aligned}
$$

Let boundary equilibria $E_{i}, 3 \leq i \leq 10$ be globally asymptotically stable in their respective subsystems. Then

$$
\begin{aligned}
g_{1}(0)-\overline{y_{11}} p_{1_{x_{1}}}(0,0)>0, & g_{1}(0)-\widetilde{y_{22}} q_{1 x_{1}}(0,0)>0, \\
g_{2}(0)-\widetilde{y_{2}} q_{2_{x_{2}}}(0,0)>0, & g_{2}(0)-\overline{y_{1}} p_{2_{x_{2}}}(0,0)>0, \\
-s_{2}(0)+c_{2} q_{1}\left(\overline{x_{1}}, \overline{y_{1}}\right)>0, & -s_{2}(0)+\widetilde{c_{2}} q_{2}\left(\overline{x_{2}}, \overline{y_{11}}\right)>0, \\
-s_{1}(0)+c_{1} p_{1}\left(\widetilde{x_{1}}, \widetilde{y_{2}}\right)>0, & -s_{1}(0)+\widetilde{c_{1}} p_{2}\left(\widetilde{x_{2}}, \widetilde{y_{22}}\right)>0,
\end{aligned}
$$

Recall from section 3.2

$$
\begin{aligned}
& \xi_{1}=-s_{2}(0)+c_{2} q_{1}\left(x_{31}, y_{31}\right)+\widetilde{c_{2}} q_{2}\left(x_{32}, y_{31}\right), \\
& \xi_{2}=-s_{1}(0)+c_{1} p_{1}\left(x_{41}, y_{42}\right)+\widetilde{c_{1}} p_{2}\left(x_{42}, y_{42}\right), \\
& \xi_{3}=g_{2}(0)-y_{51} p_{2_{x_{2}}}\left(0, y_{52}\right)-y_{52} q_{2_{x_{2}}}\left(0, y_{51}\right), \\
& \text { and } \\
& \xi_{4}=g_{1}(0)-y_{61} p_{1_{x_{1}}}\left(0, y_{62}\right)-y_{62} q_{1_{1_{1}}}\left(0, y_{61}\right) .
\end{aligned}
$$


For positive small $\delta$ and $\mu$ (6.1) holds.

From conditions (3) and (4) equalities (6.2) and (6.3) above hold.

When $\xi_{i}>0,1 \leq i \leq 4$, using (7), inequalities (6.4) - (6.11) hold.

We thus have the following results:

Theorem 9: Let hypotheses $\left(H_{1}\right)-\left(H_{5}\right)$ hold and condition of facultative mutualism hold. Further $E_{i}, 3 \leq i \leq 10$ exist and be globally stable in their respective octants. When $\xi_{i}>0,1 \leq i \leq 4$, system (1) will be uniformly persistent.

Theorem 10: Let hypotheses $\left(H_{1}\right)-\left(H_{5}\right)$ hold and mutualism be obligate for $y_{1}$ (i.e. condition (5.1) holds). Furthermore let $E_{5}, E_{6}, E_{i}, 8 \leq i \leq 10$ be globally stable in their respective octants. When $\xi_{i}>0,2 \leq i \leq 4$, system (1) will be uniformly persistent.

In next result we give conditions for uniform persistence when mutualism is obligate for $y_{2}$.

Theorem 11: Let hypotheses $\left(H_{1}\right)-\left(H_{5}\right)$ hold and mutualism be obligate for $y_{2}$ (i.e. condition (5.2) holds). Further let $E_{3}, E_{4}, E_{7}, E_{9}$ and $E_{10}$ be globally stable in their respective positive octants. When $\xi_{1}>0, \xi_{3}>0$ and $\xi_{4}>0$, system (1) is uniformly persistent.

When mutualism is obligate for both $y_{1}$ and $y_{2}$, equilibria $E_{i}, 3 \leq i \leq 8$ do not exist, and we have:

Theorem 12: Let hypotheses $\left(H_{1}\right)-\left(H_{5}\right)$ hold. Further let mutualism is obligate for both $y_{1}$ and $y_{2}$ (i.e. (5.1) and (5.2) hold). Let $E_{9}$ and $E_{10}$ be globally stable in $R_{x_{1} y_{1} y_{2}}^{+}$ and $R_{x_{2} y_{1} y_{2}}^{+}$, respectively. If $\xi_{3}>0$ and $\xi_{4}>0$ then system is uniformly persistent.

\section{Special case}

Finally we present a special case of model (1):

We consider the system

$$
\begin{aligned}
\frac{d x_{1}}{d t} & =a_{1}\left(1-\frac{x_{1}}{K_{1}}\right) x_{1}-p_{1}\left(x_{1}, y_{2}\right) y_{1}-q_{1}\left(x_{1}, y_{1}\right) y_{2} \\
\frac{d x_{2}}{d t} & =a_{2}\left(1-\frac{x_{2}}{K_{2}}\right) x_{2}-p_{2}\left(x_{2}, y_{2}\right) y_{1}-q_{2}\left(x_{2}, y_{1}\right) y_{2} \\
\frac{d y_{1}}{d t} & =y_{1}\left\{-s_{11}-s_{12} y_{1}+c_{1} p_{1}\left(x_{1}, y_{2}\right)+\widetilde{c_{1}} p_{2}\left(x_{2}, y_{2}\right)\right\} \\
\frac{d y_{2}}{d t} & =y_{2}\left\{-s_{21}-s_{22} y_{2}+c_{2} q_{1}\left(x_{1}, y_{1}\right)+\widetilde{c_{2}} q_{2}\left(x_{2}, y_{1}\right)\right\}
\end{aligned}
$$

For global stability of all equilibria, below $\left(\operatorname{except} E_{8}\left(x_{41}, x_{42}, 0, y_{42}\right)\right)$ we take $p_{i}\left(x_{i}, y_{2}\right)=p_{i 1} x_{i}, q_{i}\left(x_{i}, y_{1}\right)=q_{i 1} x_{i} y_{1}, i=1,2$. 
For $E_{8}$, we set:

$p_{i}\left(x_{i}, y_{2}\right)=p_{i 1} x_{i} y_{2}, \quad q_{i}\left(x_{i}, y_{1}\right)=q_{i 1} x_{i}, i=1,2$.

The model considered exhibits commensalism between $y_{1}$ and $y_{2}$.

A set of sufficient conditions for existence of $E_{i}, 7 \leq i \leq 10$ is that the corresponding submodel be uniformly persistent [ 2 ]. The constants $L_{i}, G_{i}, i=1,2$, are as in Theorem 2. Computing matrix $\mathrm{M}$ as in Theorem 4, we get

$$
M=\left[\begin{array}{ccc}
-\frac{a_{1}}{K_{1}} & 0 & \frac{\left(c_{1}-1\right) p_{11}}{2} \\
0 & -\frac{a_{2}}{K_{2}} & \frac{\left(\widetilde{c_{1}}-1\right) p_{21}}{2} \\
\frac{\left(c_{1}-1\right) p_{11}}{2} & \frac{\left(\widetilde{c_{1}}-1\right) p_{21}}{2} & -s_{12}
\end{array}\right]
$$

Corollary 1: $E_{7}$ will be globally stable in $R_{x_{1} x_{2} y_{1}}^{+}$, whenever $M$ is negative definite.

For $E_{8}$, we get

$$
N=\left[\begin{array}{ccc}
-\frac{a_{1}}{K_{1}} & 0 & \frac{q_{11}\left(c_{2}-1\right)}{2} \\
0 & -\frac{a_{2}}{K_{2}} & \frac{q_{21}\left(\widetilde{c_{2}}-1\right)}{2} \\
\frac{q_{11}\left(c_{2}-1\right)}{2} & \frac{q_{21}\left(\widetilde{c_{2}}-1\right)}{2} & -s_{22}
\end{array}\right]
$$

By Theorem 5, we have:

Corollary 2: When $N$ is negative definite, $E_{8}$ is globally stable in $R_{x_{1} x_{2} y_{2}}^{+}$.

Next let $E_{9}\left(x_{51}, 0, y_{51}, y_{52}\right)$ exist.

Computing symmetric matrix $R$ as in Theorem 6,

$R=\left(r_{i j}\right)$, is given by

$r_{11}=-\frac{a_{1}}{K_{1}}, r_{12}\left(y_{2}\right)=\frac{1}{2}\left\{\left(c_{1}-1\right) p_{11}-q_{11} y_{2}\right\}$,

$r_{13}\left(y_{1}\right)=\frac{1}{2} q_{11}\left(y_{1}-y_{51}\right), r_{22}=-s_{12}, r_{23}=\frac{1}{2} q_{11} c_{2} x_{51}$,

$r_{33}=-s_{22},\left|r_{12}(0)\right|=\frac{p_{11}}{2}\left|c_{1}-1\right|$,

Now, proceeding as in Theorem 2 of section 2,

$y_{1} \leq \delta_{19}=\frac{c_{1} G_{1}}{s_{1}(0)}+\epsilon$, for large $t, y_{2} \leq \delta_{29}=\frac{c_{2} L_{1}}{s_{2}(0)}+\epsilon$, for large $t$. 
Thus,

$\left|r_{12}\left(\delta_{29}\right)\right|=\frac{1}{2}\left|p_{11}\left(c_{1}-1\right)-q_{11} \delta_{29}\right|, \quad r_{12} \max =\max \left\{\left|r_{12}(0)\right|,\left|r_{12}\left(\delta_{29}\right)\right|\right\}$

$\left|r_{13}\left(y_{1}\right)\right| \leq \frac{q_{11}}{2} \max \left\{y_{51},\left|\delta_{19}-y_{51}\right|\right\}=r_{13} \max$

Corollary 3: Let $E_{9}\left(x_{51}, 0, y_{51}, y_{52}\right)$ exists and

$$
\frac{a_{1}}{K_{1}}>r_{12} \max +r_{13} \max , s_{12}>r_{12} \max +r_{23}, s_{22}>r_{13} \max +r_{23} \text {. }
$$

Then $E_{9}$ is globally stable in $R_{x_{1} y_{1} y_{2}}^{+}$.

\section{Proof:}

Under condition(12), $R$ is a symmetric diagonally dominant matrix in $\mathcal{A}_{3}$. By Gersgorin theorem if $\lambda$ is an eigenvalue then there exists $1 \leq i \leq 3$ such that

$\lambda \leq r_{i i}+\sum_{j \neq i}\left|r_{i j}\right|$

Also $\left|r_{i i}\right|>\sum_{k \neq i}\left|r_{i k}\right|$ and $r_{i i}<0, j=1,2,3$. Thus, $r_{i i}+\sum_{j \neq i}\left|r_{i j}\right|<0$

i.e. eigenvalues of $R$ in $\mathcal{A}_{3}$ are negative and by Theorem $6 E_{9}$ is globally stable in $R_{x_{1} y_{1} y_{2}}^{+}$

Next, let $E_{10}\left(0, x_{62}, y_{61}, y_{62}\right)$ exists. From Theorem 2, $y_{i}(t) \leq \delta_{i 10}$ for large $\mathrm{t},=1,2$. where,

$\delta_{110}=\frac{\widetilde{c_{1}} G_{2}}{s_{11}}+\epsilon, \delta_{210}=\frac{\widetilde{c_{2}} L_{2}}{s_{21}}+\epsilon$,

Computing symmetric matrix $T$, as in Theorem 7 , then

$T=\left(t_{i j}\right)_{3 \times 3}$

where,

$t_{11}=-\frac{a_{2}}{K_{2}}, t_{12}\left(y_{2}\right)=\frac{1}{2}\left\{p_{21}\left(\widetilde{c_{1}}-1\right)-q_{21} y_{2}\right\}, t_{13}=\frac{1}{2} q_{21}\left(y_{1}-y_{61}\right), t_{22}=-s_{12}$,

$t_{23}=\frac{1}{2}\left[q_{21} \widetilde{c_{2}} x_{62}\right], t_{33}=-s_{22}$,

and

$t_{i j}=t_{j i}, i>j$. 
Define,

$t_{12} \max =\frac{1}{2} \max \left\{\left|t_{12}(0)\right|, t_{12}\left(\delta_{210}\right)\right\}, t_{13} \max =\frac{1}{2} \max \left\{q_{21} y_{61}, q_{21}\left(\delta_{10}-y_{61}\right)\right\}$

We require

$\frac{a_{2}}{K_{2}}>t_{12} \max +t_{13} \max , s_{12}>t_{12} \max +t_{23}, s_{22}>t_{13} \max +$

$t_{23}$.

Then we have the following result:

Corollary 4: Let $E_{10}\left(0, x_{62}, y_{61}, y_{62}\right)$ exists and inequalities (13) hold.

Then $E_{10}$ is globally asymptotically stable in $R_{x_{2}}^{+} y_{1} y_{2}$.

For global stability of $E^{*}$, we computing $L$, as in Theorem 8 ,

$$
\begin{aligned}
& u_{11}=-\frac{a_{1}}{K_{1}}, u_{12}=0, u_{13}=\frac{1}{2}\left\{\left(c_{1}-1\right) p_{11}-q_{11} y_{2}\right\}, u_{14}=\frac{1}{2} q_{11}\left(y_{1}-y_{1}^{*}\right), \\
& u_{22}=-\frac{a_{2}}{K_{2}}, u_{23}=\frac{1}{2}\left\{\left(\widetilde{c_{1}}-1\right) p_{21}-q_{21} y_{2}\right\}, u_{24}=\frac{1}{2} q_{21}\left(\widetilde{c_{2}} y_{1}-y_{1}^{*}\right), u_{33}=-s_{12}, \\
& u_{34}=\frac{1}{2}\left(c_{2} x_{1}^{*} q_{11}+\widetilde{c_{2}} x_{2}^{*} q_{21}\right), u_{44}=-s_{22} . \\
& u_{i j}=u_{j i}, i>j, 1 \leq i, j \leq 4 . \\
& \text { Set } \mathrm{U}=\left(u_{i j}\right) .
\end{aligned}
$$

Next, if $E^{*}\left(x_{1}^{*}, x_{2}^{*}, y_{1}^{*}, y_{2}^{*}\right)$ exists in interior of $R_{x_{1} x_{2} y_{1} y_{2}}^{+}$, Define

$l_{13} \max =\frac{1}{2} \max \left\{\left|\left(c_{1}-1\right) p_{11}\right|,\left|\left(c_{1}-1\right) p_{11}-q_{11} \delta_{2}\right|\right\}, l_{14} \max =\frac{1}{2} q_{11} \max \left\{\left(\delta_{1}-\right.\right.$ $\left.\left.y_{1}^{*}\right), y_{1}^{*}\right\}$,

$l_{23} \max =\frac{1}{2} \max \left\{\left|\widetilde{c_{1}}-1\right| p_{21},\left|\left(\widetilde{c_{1}}-1\right) p_{21}-q_{21} \delta_{2}\right|\right\}, l_{24} \max =\frac{1}{2} q_{21}\left(\widetilde{c_{2}} \delta_{1}-y_{1}^{*}\right)$,

where, $\delta_{1}=\frac{c_{1} G_{1}+\widetilde{c_{1}} G_{2}}{s_{11}}$, and $\delta_{2}=\frac{c_{2} L_{1}+\widetilde{c_{1}} L_{2}}{s_{21}}$.

The next result is clear from Theorem 8 .

Corollary 5: Let $E^{*}\left(x_{1}^{*}, x_{2}^{*}, y_{1}^{*}, y_{2}^{*}\right)$ exists and

$\frac{a_{1}}{K_{1}}>l_{13} \max +l_{14} \max , \frac{a_{2}}{K_{2}}>l_{23} \max +l_{24} \max$,

$s_{12}>l_{13} \max +l_{23} \max +l_{24} \max$, and $s_{22}>l_{14} \max +l_{24} \max +l_{34}$.

Then $E^{*}$ is globally stable.

Below we present specific examples:

With $a_{1}=a_{2}=1, K_{1}=0.8, K_{2}=1, p_{11}=.4, p_{21}=.3, c_{1}=2.5, \widetilde{c_{1}}=3.5$, 
$s_{11}=.5, s_{12}=1.5, E_{7}\left(x_{31}, x_{32}, y_{31}, 0\right)=(0.5977,0.8103,0.6323,0)$ exists and the symmetric matrix

$$
M=\left[\begin{array}{ccc}
-1.25 & 0 & .3 \\
0 & -1 & .375 \\
0.3 & .375 & -1.5
\end{array}\right]
$$

is negative definite as its eigenvalues are $-1.8263,-1.1716,-.7521$. Hence by Theorem $4 E_{7}$ is globally stable in $R_{x_{1} x_{2} y_{1}}^{+}$.

With $a_{1}=a_{2}=1, K_{1}=K_{2}=1, q_{11}=0.4, c_{2}=2.5, \widetilde{c_{2}}=3.5, q_{21}=0.3, s_{21}=$ 0.5 and $s_{22}=1.50$.

Equilibrium $E_{8}\left(x_{41}, x_{42}, 0, y_{41}\right)=(0.7201,0.7901,0,0.6998)$ exists and symmetric matrix $N$ in Theorem 5 is

$$
N=\left[\begin{array}{ccc}
-1 & 0 & .3 \\
0 & -1 & .3750 \\
0.3 & .375 & -1.5
\end{array}\right]
$$

and has eigenvalues $-1.7914,-1$ and -0.7086 . Thus $E_{8}$ is globally stable in $R_{x_{1} x_{2} y_{2}}^{+}$

With $a_{1}=2.2, K_{1}=0.85, p_{11}=0.45, q_{11}=.21, s_{11}=0.5, s_{12}=2.52$,

$$
s_{21}=0.065, s_{22}=1.75, c_{1}=2.4 \text { and } c_{2}=3.47, E_{9}\left(x_{51}, 0, y_{51}, y_{52}\right)=
$$
$(0.823,0,0.1543,0.0157)$ exists and conditions (12) of Corollary 3 are satisfied. Thus $E_{9}$ is globally stable in $R_{x_{1} y_{1} y_{2}}^{+}$.

With $a_{2}=1.8, K_{2}=0.80, p_{11}=0.4, p_{21}=.42, q_{21}=.25, s_{11}=0.50$, $s_{12}=1.9, s_{21}=0.1, s_{22}=1, \widetilde{c_{1}}=2.55$ and $\widetilde{c_{2}}=3.5, E_{10}\left(0, x_{62}, y_{61}, y_{62}\right)=$ $(0,0.768,0.1698,0.0141)$ exists. Also conditions of Corollary 4 are satisfied and $E_{10}$ is globally stable in $R_{x_{2} y_{1} y_{2}}^{+}$.

With $a_{2}=1, K_{1}=2, a_{2}=1.05, K_{2}=1, p_{11}=0.365, q_{11}=0.25, p_{21}=.30$, $q_{21}=0.5, s_{11}=0.4, s_{12}=0.37, s_{21}=0.4, s_{22}=0.90, c_{1}=1, \widetilde{c_{1}}=1.5, c_{2}=$ 1.2 , and $\widetilde{c_{2}}=2.5$, interior equilibrium $E^{*}\left(x_{1}^{*}, x_{2}^{*}, y_{1}^{*}, y_{2}^{*}\right)=$ $(1.2352,0.7106,1.0017,0.0669)$ exists. The conditions of Corollary 5 are also satisfied and $E^{*}$ is globally stable.

Next we illustrate Theorem 11 .

With $a_{1}=1.8, a_{2}=1.8, K_{1}=0.8, K_{2}=0.8, p_{11}=0.4, p_{21}=.42, s_{11}=0.5$, $s_{12}=1.9, s_{21}=0.1, s_{22}=1, \widetilde{c_{1}}=2.55, c_{2}=3.5, \widetilde{c_{2}}=3.5, c_{2}=3.5, \widetilde{c_{2}}=$ $3.5, q_{11}=0.25$ and $q_{21}=0.25$, equilibria $E_{0}(0,0,0,0), E_{1}(0.8,0,0,0)$, $E_{2}(0,0.8,0,0), E_{3}(0.4902,0,3.3971,0), E_{4}(0,0.3401,1.7847,0)$, $E_{7}(0.7086,0.7040,0.5140,0), E_{9}(0.7730,0,0.1518,0.0027)$ and 
$E_{10}(0,0.7680,0.1698,0.0141)$ exist. Further $E_{3}, E_{4}, E_{7}, E_{9}$ and $E_{10}$ are globally stable in their respective octants. Finally, $\xi_{1}=0.5355, \xi_{3}=1.7361, \xi_{4}=1.7315$. are positive and by Theorem 11 system (9) with $p_{i}, q_{i}$ as given by (10) is uniformly persistent.

\section{REFERENCES}

[1] S. Ahmad and M.R.M. Rao, Ordinary Differential Equations, Affiliated East West Press Private Ltd. (2014).

[2] G. J. Butler, H.I. Freedman and P. Waltman Uniformly Persistent Systems, Proc. Amer. Math. Soc. 96, 425 - 430(1986).

[3] H .I. Freedman, R. Kumar, A.K. Easton and M. Singh, Mathematical Models of Predator Mutualists, Canadian Applied Mathematics Quarterly, Vol. 9, 99 - 111 (2001).

[4] B. S. Goh, Management and analysis of biological populations, Elsevier (1980).

[5] P. Hartman, Ordinary differential equation, Birkhauser, second edition(1980).

[6] V. Hutson, A Theorem on Average Functions, Mh. Math. 98: 267 - 275 (1984).

[7] R. Kumar and H. I. Freedman, A mathematical model of facultative mutualism with population interacting in a food chain. Math. Biosci. 97 : 235 - 261 (1989).

[8] R. Ormond and A. J. Edwards, Red Sea Fishes, in Red Sea (A. J. Edwards and S. M. Head, eds.). Pergamon, New York, 1987.

[9] B. Rai, P. Khare and M. Singh, Mutualistic Interactions Leading to Coexistence in Competitive Ecological Communities, Jour. of Int. Academy of Physical Science $17: 325-354$ (2013).

[10] B.Rai and M. Singh, Indirect mutualism and Coexistence in Multispecies Ecological Communitities, Indian Jour. Of Industrial and Applied Maths. 4(1) $52-70$ (2013).

[11] G.S.K. Wolkowicz and Z. Lu, Global Dynamics of a Math. Model of Competition in the chemostat: General respone functions and differential death rates, SIAM J. Appl. Math. 52: 222 - 233 (1992). 